\title{
Estudio de la compensación a las pérdidas en la traducción chino-español de los nombres de los platillos chinos
}

\section{Research on Compensation for Losses in the Chinese- Spanish Translation of the Name of Chinese Dishes}

\author{
DOI: $10.32870 /$ mycp.v8i24.612
}

Jie Zhou ${ }^{1}$

\begin{abstract}
Resumen
Es del dominio común que la cocina china se cuenta entre las más conocidas del mundo, y que el simple hecho de comer forma parte inseparable de la vida cotidiana. Razón por la cual la comida china supone una atracción para los turistas provenientes de diferentes continentes y los interesados en la cultura milenaria de China. Sin embargo, debido a la variedad de los ingredientes y las formas de cocinar, así como las disparidades culturales y lingüísticas, inevitablemente surgen pérdidas durante el proceso de traducción de los nombres de los platillos chinos. A fin de conservar lo más posible la esencia de los platillos chinos, la traducción de éstos merece profundos estudios, tema que tratamos de analizar desde el ángulo chino-español.

El presente estudio se articula en tres partes. En la primera se presentan algunas teorías generales de las pérdidas y la compensación de traducción. Se abordan en la segunda parte la denominación de los platillos chinos y los posibles factores que ocasionan pérdidas en la traducción. La tercera parte se desarrolla en torno a la compensación que se puede aplicar en este proceso, ilustrándose con diversos ejemplos.
\end{abstract}

Palabras clave: traducción chino-español, platillos chinos, pérdida, compensación, interculturalidad.

\begin{abstract}
It is common knowledge that Chinese cuisine is among the best known of the world, and the simple fact of eating is an inseparable part of daily life. That is why Chinese food attracts tourists from different continents, who are interested in the millenary culture of China. However, due to the variety of ingredients and ways of cooking, as well as cultural and linguistic disparities, losses would inevitably arise during the process of translating the names of Chinese dishes. In order to keep the essence of Chinese dishes as real as possible, the translation of these deserves profound studies. This is a subject that we try to analyze translation from the Chinese-Spanish angle. This paper is divided into three parts. Firstly, some general theories of losses and translation compensations would be presented. The name of the Chinese dishes and the possible factors that cause translation losses would be addressed in the second part. The third part is developed around the compensations that could be applied in this process, illustrated with several examples.
\end{abstract}

Keywords: Chinese-Spanish translation, Chinese dishes, translation loss, compensation, interculturality.

Artículo recibido el 11 de enero de 2019 y dictaminado el 30 de abril de 2019.

1. Shanghai International Studies University (SISU), 550 Dalian West Road, Hongkou District, 200083, Shanghai, China. ORCID: http://orcid.org/0000-0002-6966-3393. Correo electrónico: jzhou@shisu.edu.cn 


\section{Introducción}

Es bien sabido que la gastronomía china goza de mucha fama en todo el mundo por su variedad y exquisitez. Los nombres de los platillos suelen contener ciertos sentidos culturales, por lo que la traducción de esos mismos desempeña un papel imprescindible para la difusión de la cultura gastronómica y que los lectores experimenten la esplendidez de la cocina.

Pese a que los nombres de los platillos chinos están compuestos solamente de unos cuatro caracteres, a veces el trabajo de traducción no resulta nada fácil debido a diversas causas, tales como las diferencias culturales y lingüísticas, o la incapacidad del traductor. La cocina es una cultura que encierra ciertos factores que, pese al esfuerzo que pongamos, posiblemente no podamos encontrar la correspondencia en la lengua de destino; por ejemplo, los ingredientes, la historia a la que se hace referencia, e incluso los sabores. No nos faltan ejemplos al respecto que motivan las pérdidas durante el proceso de la traducción de los nombres de los platillos chinos.

Naturalmente, la necesidad de compensación traductora se agudiza frente a las pérdidas inevitables. Es indispensable para nosotros procurar que, al compensar las pérdidas de una u otra manera, la información se transmita lo más fiel y lo más natural posible y que los destinatarios de la traducción reaccionen como los lectores del idioma chino.

\section{Pérdidas y compensación de traducción}

La traducibilidad y la intraducibilidad siempre han sido una controversia incesable. A pesar de que cuanto más lejanos están dos idiomas y menos contactos mantienen, se observan entre sí más disparidades, tal es el caso de la familia lingüística indoeuropea y la sino-tibetana; preferiríamos decir que la intraducibilidad es relativa y la traducibilidad, a su vez, tiene sus límites, lo cual da origen a las pérdidas y es la razón por la que existe la compensación.

¿Por qué deberíamos compensar a lo largo del proceso de traducción? La respuesta será sencilla: cumplimos el acto de compensar puesto que aparecen las pérdidas que se reflejan en la información, el contenido semántico, factores culturales, los efectos pragmáticos, estilísticos, estéticos, etcétera.

Según indica el lingüista y traductólogo Eugene A. Nida: "En la comunicación verbal siempre surge algo de pérdida y distorsión ya que no existen dos interlocutores que tengan exactamente el mismo significado denotativo y 
asociativo para los mismos rasgos fonológicos, léxicos, gramaticales y discursivos" (2001). Por lo tanto, se puede afirmar que las pérdidas surgen inevitablemente y que nuestra actividad traductora va acompañada de las mismas. El objetivo buscado por los traductores consiste en presentar al lector una versión con fidelidad, pero a veces terminamos el trabajo sin darnos cuenta de que se desvanecen algunos elementos bastante relevantes: la información cultural, el valor estético del texto original, entre otros.

Nos podemos imaginar que en la traducción chino-español las pérdidas son numerosas porque entre dichos dos idiomas no hay parentesco. No obstante, el hecho de que las pérdidas se produzcan no sólo se debe a las causas objetivas, como las características fonológicas, morfológicas, sintácticas o culturales, sino también a las subjetivas, esto es, la incapacidad del traductor, que abarca la carencia del dominio de las dos lenguas y la influencia de la lengua materna y del entorno social y cultural que le rodea.

En su obra En torno a la traducción, Valentín García Yebra, eminente filólogo y traductor español, define así la traducción: "Es un proceso lingüístico mediante el cual se reproduce en la lengua receptora el mensaje de la lengua original" (1989). Con el fin de que los lectores de la lengua terminal reaccionen como los del texto de partida, a veces es imprescindible compensar las pérdidas tomando medidas adecuadas. Lucía Molina señala que la compensación consiste en "Introducir en otro lugar del texto meta un elemento de información o efecto estilístico que no se ha podido reflejar en el mismo lugar en que aparece situado en el texto original" (Molina, 2006). En otras palabras, se trata de un trabajo que se esfuerza dentro de lo posible por pasar los contenidos del texto original, tanto semánticos como estilísticos, a la lengua de llegada. En definitiva, los dos traductólogos, a quienes daríamos la razón, llegan al acuerdo de que la compensación tiene como objetivo tratar de conseguir que se genere un efecto similar.

Sobre la base de la teoría y a fin de evitar consecuencias negativas y mantener ingredientes positivos, aquí plantearíamos algunos principios a los que debe ajustarse la compensación (citado en Xia, 2006, p. 191).

- Principio de demanda. Como lo que hemos mencionado, en muchos casos las pérdidas se producen de manera inevitable. Sin embargo se recomienda la compensación solamente cuando los contenidos perdidos cumplen un papel clave en la comprensión de los lectores de la lengua de destino, tal es el caso de traducir el platillo “龙虎斗”: la versión literal "lucha entre dragón y tigre” será una traducción mala, ya que es obligatorio 
aclarar su significado metafórico después de presentar la traducción literal, especificando que el platillo trata de una sopa de serpiente y gatoleopardo, ${ }^{2}$ con el fin de que los comensales se enteren de qué van a probar.

- Principio de enfoque. Se concede primacía a los contenidos perdidos más relevantes cuando surgen dos o más pérdidas. Este principio nos exige distinguir bien cuál es la parte prioritaria donde hace falta la compensación. En las partes posteriores lo examinaremos con más profundidad, ilustrándonos con ejemplos.

- Principio de igual función. Este principio dice que no importa si se aprovechan los medios específicos iguales que el texto original, y lo que importa es si la compensación obtiene un efecto equivalente. Esto es, la meta de la compensación a la traducción de los nombres de los platillos consiste en conseguir que los lectores de la lengua terminal sepan a primera vista de qué trata el platillo. En lugar de traducir literalmente “佛跳墙” como "el monje saltando el muro", conservando la alusión que encierra, solemos añadir aclarando que en realidad es un platillo representativo de Fujian, elaborado con unos 18 ingredientes.

\section{Denominación de los platillos chinos}

Será imposible encontrar un platillo sin nombre en la cocina china, puesto que la denominación no sólo significa un código de la comida, sino que también encarna la esencia de la misma y constituye un estudio que merece muchas investigaciones. Con base en las teorías mencionadas en los apartados anteriores, aclararemos en este apartado diversas formas de denominar los platillos y distintos elementos que motivan la falta de claridad, los malentendidos, la disminución de los valores estilísticos o estéticos, etc., lo cual constituye un tema que vale la pena dejar claro antes de adentrarnos en la aplicación de la compensación.

Denominación según ingredientes. Una gran cantidad de los platillos chinos adquieren sus nombres de acuerdo con los ingredientes que emplean. De esta forma, se facilita en gran medida la comprensión de los comensales

2. Platillo del estilo culinario cantonés. No es de demanda común en China, donde casi no se consumen los platillos con gato. La serpiente es un ingrediente para la medicina china, y sí se ofrece en algunos menús. 
de qué alimentos van a probar en el platillo que se servirá. No nos faltan ejemplos de este tipo: “木耳肉片” (traducción literal: muer y rebanada de carne), “酸菜鱼” (col fermentada y pescado), “荷叶鸡” (hojas de loto y pollo), “冰糖燕窝” (azúcar piedra y nido de salangana), “糯米鸭” (arroz glutinoso y pato), entre otros.

Denominación según color, aroma y sabor. Todo el mundo sabe que el color, el aroma y el sabor constituyen tres puntos fundamentales que cuida mucho la gastronomía china. Con los diversos casos nos damos cuenta de que existen una buena parte de platillos que se identifican denominándose según dichos puntos. Tomemos como ejemplo “琥珀肉” (traducción literal: carne de ámbar), “五香肉” (carne de cinco aromas), “鱼香肉丝” (tiritas de carne con sabor de pescado), que sacan la característica más representativa, o sea, el color, el aroma y el sabor, respectivamente, de esos platillos a fin de añadir su encanto y excitar el apetito.

Denominación según formas de cocinar. Es digno de señalar que, aparte de lo arriba mencionado, la comida china también se caracteriza por la variedad de las numerosas formas de cocinar, como por ejemplo: saltear, hervir, freír, sofreír, refreír, asar, guisar, estofar, ahumar, salar, cocer al vapor, con salsa de soya, a la cazuela, al caldero mongol, etc., según las cuales surge naturalmente la denominación de abundantes platillos. Para mayor claridad, ahora vamos a ejemplificarlo con “粉蒸牛肉” (traducción literal: ternera cocida a vapor con harina), “红烧肉” (carne cocida son salsa de soya), “酱猪蹄” (patas de cerdo sazonadas con salsa), “干煸鳔鱼” (anguila frita), y “火爆鸭肠” (intestinos de pato fritos), a través de los cuales se transmite cómo se elaboran dichos alimentos suculentos.

Denominación según alusiones. Inspirándonos en nuestra experiencia y prácticas de traducción, podríamos llegar a la conclusión de que la medida principal y más usada que generalmente se adopta en la denominación de los platillos chinos es la forma realista, que hace patente la composición de la materia prima, las formas de cocinar, así como sus características típicas. Sin embargo, no se puede negar que se halle una buena parte de los platillos chinos que logran sus nombres aprovechando la cultura, la historia, la literatura, e incluso las figuras retóricas.

Como se ha discutido, denominar conforme a las alusiones también constituye una forma común que no podemos pasar por alto. Se ve que los platillos que se denominan por medio de los nombres de sus creadores, por ejemplo “麻婆豆腐” (traducción literal: tofu de la abuela Mapo), “东 
坡肉” (carne hecha por el poeta Su Dongpo) tienen como objetivo rendir homenaje a ellos, en este caso a la abuela Mapo y al poeta Su Dongpo. Además, algunos nombres de los platillos chinos implican un suceso que tiene lugar en cierto momento a lo largo de la historia. Los ejemplos siguientes son de este tipo: el bocadillo “油炸鬼” (literalmente “fantasma refreído"), nombre del churro chino que todavía se emplea en gran escala en las provincias del sur, lleva en sí mismo el odio de la gente común de la dinastía Song por Qin Hui, el cortesano más traicionero de esa época; la alusión que contiene el famoso platillo “贵妃鸡翅” (literalmente "alitas de pollo favoritas de la concubina") se remonta a la era dinástica Tang, ya que los cocineros de la familia real inventaron este maravilloso platillo de acuerdo con el gusto de la concubina Yang Yuhuan, una de las cuatro bellas de la China antigua. En fin, son abundantes los ejemplos; aquí no vamos a enumerarlos uno por uno.

Denominación de forma metafórica. Realzando la sensación estética de los nombres de los platillos chinos durante el proceso cognitivo del lector, se utiliza universalmente la metáfora al denominarlos, con lo cual se dejará una impresión más profunda y se transmitirá con mayor facilidad la presentación de un platillo. Véanse los siguientes ejemplos. El nombre “珍珠豆腐” (literalmente “tofu con perlas") no significa que en medio del tofu haya verdaderas perlas, sino que el tofu es tan brillante y blanco que se parece a las perlas. Lo mismo ocurre con el nombre “虎皮青椒” (pimientas con piel del tigre). En él no se toma, sin duda alguna, la piel de los tigres como ingrediente, sino que los pimientos cocidos parecen llevar una capa de la piel de los tigres. No es un caso menos exitoso el “蚂蚁上 树”, que literalmente se refiere a "hormigas sobre el árbol”, cuyo significado metafórico consiste en que los picados de carne están salpicados por los fideos como si fueran hormigas que intentan trepar a un árbol. Otros ejemplos: “红烧狮子头” (cabeza de león cocida con salsa de soya), “掌上 明珠”(perlas en la palma), etcétera.

Debido al acelerado desarrollo de la gastronomía y el límite de espacio, nos reducimos a aclarar las cinco formas de la denominación. 


\section{Factores que ocasionan pérdidas en la traducción}

Si tratamos de llevar a cabo una versión lo más fiel y lo más natural posible, no nos basta con dominar la gramática, el vocabulario y la capacidad oral y escrita, puesto que se exige a los traductores, en la mayoría de los casos, averiguar con profundidad las culturas de ambas lenguas con el fin de llegar a una equivalencia dinámica que atiende, "más que a la forma del mensaje, a la reacción de sus receptores porque lo que se busca en este 'nuevo' modo de traducir es que los destinatarios de la traducción reaccionen, en lo posible, como los lectores u oyentes del texto original" (García, 1989).

Como la lengua es vehículo de la cultura, es difícil encontrar palabras con significados completamente equivalentes a todo nivel en diferentes culturas. A fin de ilustrarnos, tomemos el ejemplo de la palabra "pavo". Muy posiblemente lo que se les ocurrirá primero a los lectores resultará el Día de Acción de Gracias, mientras que los chinos, como no suelen comerlo, lo tomarán solamente como un animal comestible, sin asociarlo con nada cultural. En definitiva, el fenómeno arriba mencionado nos advierte tener en cuenta el aspecto cultural de la lengua traducida y no poner siempre en un lugar prioritario la cultura de la lengua de salida para llegar a una equivalencia funcional en plano cultural.

Disparidades geográficas. Gracias a la vasta extensión de China, se notan entre las diversas zonas no pocas diferencias en cuanto a la gastronomía. En primer lugar, se trata de una práctica general tomar los productos de harina (pan chino, tallarines, ravioles chinos, etc.) como la comida principal en las provincias del norte, a diferencia de los sureños, que suelen comer arroz. En segundo lugar, hay en total ocho grandes estilos culinarios en toda China y se diferencian unos de otros. Por ejemplo, el de Cantón se destaca por la frescura y el sabor natural de los alimentos, mientras que el de Sichuan usa muchas especies y mucha grasa, tirando a sabores picantes, por lo que un mismo platillo puede resultar diferente en ingredientes y sabores, dependiendo del gusto y de la cadena de abastecimiento local. Por último, por razones de las diferencias climáticas y topográficas y el entorno particular en el que se encuentran, en ciertas localidades se hallan algunos alimentos característicos, los cuales muchas veces hacen famoso el lugar de origen; tal es el caso del cangrejo procedente del lago Yangcheng, situado en la provincia de Jiangsu.

Por todo esto, cuando se presentan delante de nosotros los platillos que se denominan con nombres geográficos, generalmente los vamos a asociar con la autenticidad y la exquisitez, considerando que no se puede encontrar 
un platillo más suculento en otro lugar. Sin embargo, durante el proceso de la traducción de los platillos que adquieren sus nombres de esta forma, la parte geográfica constituye un factor que causa pérdidas puesto que para un lector de la lengua de llegada, el pato asado no tendrá nada que ver con Beijing. A manera de ilustración, aparte del ejemplo de “北京烤鸭” (pato laqueado de Beijing) que arriba mencionamos, véanse los dos ejemplos siguientes. El “ 金华火腿” (jamón de Jinhua) tiene su origen en el municipio Jinhua de la provincia Zhejiang, el cual se convierte en afamado dentro de China gracias a la reputación que disfruta el jamón de aquí, que se caracteriza por su incomparable color, aroma, sabor y presentación. También forman parte de los ejemplos representativos “嘉兴粽子” (tamal de Jiaxing), “长沙臭豆腐” (tofu maloliente de Changsha), “贵州茅台” (licor Marotai de Guizhou), etcétera.

Disparidades históricas. A lo largo de la historia milenaria de nuestra patria se han conservado numerosísimos acontecimientos, de los cuales algunos pasan a formar parte de los nombres de los platillos chinos. De todo esto, al llevar a cabo el trabajo de traducción de los nombres pertenecientes a este tipo, las disparidades históricas se cuentan entre los factores que dan origen a las pérdidas.

Para facilitar la comprensión, generalmente traducimos “担担面” por "fideos a la sichuanesa", pero en realidad se trata de un bocadillo del cual los dos primeros caracteres “担担” contienen un cuento que nos hace recordar su origen: el nombre “担担” proviene de los bambúes que llevan los vendedores callejeros para transportar esta comida, ya que antes era un platillo muy popular que se servía fundamentalmente en la calle. Otro ejemplo es “夫妻 肺片” (literalmente “vísceras de la pareja”): la versión de “vísceras de ternera con salsa picante", a pesar de su buena aceptabilidad, no refleja la historia de la pareja que creó este platillo.

Factores gastronómicos. En el caso de la traducción de los nombres de los platillos chinos, la diferencia en el vocabulario resultará uno de los mayores obstáculos para hacer entender a los lectores. A pesar de que algunos términos culinarios son habituales en la gastronomía china, no existe su correspondencia en el español.

Tomemos unos ejemplos. En la región suroeste de China el ingrediente “花 椒”3 se emplea con frecuencia, pero no dará con una palabra correspondiente

3. Se trata de un condimento que se usa con frecuencia en la cocina china y causa una sensación similar a la parálisis en la lengua. 
en los diccionarios de la lengua terminal. En la actualidad se suele traducirlo por "pimienta china" a fin de indicar que este ingrediente procede de China y se parece a la pimienta, que llamamos “胡椒”, recuperando dentro de lo posible las pérdidas ocasionadas por la desigualdad del léxico. Lo mismo ocurre en la traducción de otras especies vegetales o animales exclusivas de nuestro país.

En cuanto a los sabores, la situación es similar. El “麻”, sabor que trae el “花椒” (pimienta china), ocupa un lugar insustituible en el paladar chino; sin embargo, sólo se puede guardar una cierta reminiscencia con la versión de "un sabor que provoca parálisis en la lengua" para los lectores que no lo han probado, puesto que no se puede hallar una palabra equivalente en el español.

Por último, pasamos a descubrir la disparidad en las formas de cocinar. Muchas veces traducimos “火爆鸭肠” como “intestinos de pato fritos". No obstante, si consultamos la definición de la palabra "freír" en el Diccionario de la Real Academia Española, nos informaremos de que significa "hacer que un alimento crudo llegue a estar en disposición de poderse comer, teniéndolo el tiempo necesario en aceite o grasa hirviendo", por lo cual la palabra "freír" no sólo se puede referir a “火爆”, sino también a “炒”, “爆” u otras formas de cocinar, y la traducción "intestinos de pato fritos" en cierta medida no refleja completamente lo que transmite el nombre chino.

La diferencia de dichos términos chinos tiene que ver con la intensidad del fuego, el tiempo de cocción, la cantidad de aceite que se usa, la cantidad de líquido que tiene, el orden de procesamiento y los ingredientes principales que se usan, entre otras variables. Por ende, podríamos llegar a la conclusión de que cuando las formas de cocinar en chino y su traducción en español no son perfectamente correspondientes, las pérdidas serán inevitables.

\section{Compensación en la traducción de los nombres de los platillos chinos}

\section{Compensación con base en la adaptación}

Los términos de clara carga cultural ocasionarán pérdidas durante la traducción. Pues bien, pueden existir situaciones comunicativas en la lengua de origen que no se ajustan absolutamente al pensamiento racional $-\mathrm{o}$, al menos, difícilmente inteligibles - en el ámbito cultural de la lengua receptora. "En tales casos, en el límite mismo de la traducibilidad, Viany y Darbelnet nos aconsejan recurrir a la adaptación, esto es, a la sustitución de la situación 
de la lengua de partida por una situación análoga de la lengua terminal, o la menos alejada posible" (Torre Esteban: 1994) a fin de realizar la compensación.

Limitándonos a hablar de la cocina china, no nos faltan ejemplos de los platillos que llevan características culturales notables: el “粽子”, “春卷”, “月 饼”, entre otros. El hecho de que dichos términos, importantes para la lengua original, no se hallen en la cultura de la lengua a la que se traduce, dará origen a lo que llamamos intraducibilidad cultural. Sin exigirlo demasiado (lo que le obliga a procurar que los lectores no se den cuenta de que están leyendo una traducción), el traductor deberá tratar de ofrecer una versión que pueda ser comprendida sin un esfuerzo extraordinario, para conseguirlo se recomienda compensar las pérdidas recurriendo en muchas ocasiones a la técnica de la adaptación.

A manera de ilustración, volvamos al ejemplo del “粽子”. Elaborado de arroz glutinoso y cocinado al vapor o hirviendo, esta comida de diferentes rellenos y envuelta con hojas de bambú no adquiere un valor, ni cultural ni simbólico, en la visión de los lectores del español ya que no existe una correspondencia equivalente en la lengua receptora. Sin embargo, si observamos la gastronomía del mundo hispanohablante, descubriremos que hay una comida que se parece al “粽子”, esto es, el tamal, bocadillo de origen indígena preparado generalmente con masa de maíz rellena de distintos ingredientes, envuelto en hojas de mazorca de maíz y cocido en agua o al vapor. Con el fin de que los lectores puedan entender los rasgos generales del “粽子” sin un gran esfuerzo, usamos “tamal” en su traducción, aprovechando la técnica de la adaptación. Y para realizar la compensación, cuando se vierte “粽子”, se añade al mismo tiempo "chino". De esta manera, se facilita en gran medida la llegada de la información a los lectores, que conocerán que el "tamal chino" contiene su propia historia y comparte unas características con el tamal mexicano.

Lo mismo ocurre en la traducción del “春卷”. Teniendo en cuenta las características de esta comida, será recomendable adoptar el "taco" como un componente del nombre traducido, puesto que sabemos que el taco es un platillo de origen mexicano que consiste en una tortilla enrollada que puede contener, de manera opcional, algún aliño dentro de ella. Para que las pérdidas ocasionadas se recuperen, lo único que nos falta consiste en completar con 
la palabra "chino" después de "taco" y de esta forma conseguiremos una traducción relativamente fiel y natural: "taco chino". ${ }^{4}$

Esta técnica también se encuentra disponible en la traducción de los siguientes términos:

“馒头”: “pan chino".

“四喜丸子: “las cuatro albóndigas chinas alegres”.

“花椒”: "pimienta china”.

“ $\square$ 子”: "raviol chino".

“油条”: "churro chino".

“月饼”: “mazapán chino".

\section{Compensación mediante amplificación y/o explicación}

A pesar de que ya tradujimos el “粽子” por “tamal chino”, en algunas ocasiones si la circunstancia demanda, es necesario explicar la tradición que encierra. En este caso, añadiremos en la traducción del chino al castellano "un platillo típico de la Fiesta de Regatas de Dragón"; esta técnica la denominamos como compensación mediante explicación. Se recomienda exteriorizar la parte que los lectores no comprenden con facilidad, utilizando la técnica traductora de amplificación a fin de ayudar a que la entiendan mejor. Según Lucía Molina, la amplificación se entiende por "introducir precisiones no formuladas en el texto original: informaciones, paráfrasis explicativas" (Molina, 2006). Al encontrarnos con un nombre de cierto platillo chino, del cual alguna parte a los extranjeros les cuesta entender y muy posiblemente impedirá la llegada de la integridad del mensaje, se suelen compensar las pérdidas por medio de esta estrategia a la hora de proceder al trabajo de traducción. Lo vamos a desarrollar a continuación con ejemplos y breves análisis:

“回锅肉”: “cerdo dos veces cocinado” (una vez cocido y la otra frito).

Si el traductor presenta la versión con la sola frase "cerdo dos veces cocinado", el lector se sentirá perplejo pensando que basta con una sola vez para que la carne quede cocida. Teniendo como objetivo acabar con sus dudas y hacerle experimentar la esencia de este maravilloso platillo del estilo culina-

4. Aunque ahora ya es bastante común la traducción "rollito de primavera". 
rio sichuanés, es imprescindible explicar aclarando que se introduce la carne en agua con sal y jengibre en la primera fase, tras ello se saca y se deja secar, para ser cortado en pequeñas rebanadas para regresar al wok y ser frita en aceite caliente.

Veamos otros ejemplos con la intención de acercarnos más a esta técnica:

“虎皮青椒”: “pimientos con piel de tigre” (la corteza de los pimientos fritos lleva muchas rayas como las de la piel del tigre).

“金银馒头”: "pan chino de oro y plata" (la combinación del pan chino frito y el cocido al vapor se presenta como si fueran oro y plata). “麻婆豆腐”: “tofu de la abuela Mapo” (tofu picante con carne picada).

Se ve que en las traducciones anteriores alcanzamos una equivalencia relativamente dinámica. Sin embargo, a veces el ingrediente de la cocina china no tiene ni la más remota contrapartida en la lengua terminal, en este caso se podrá utilizar primero la técnica de préstamo y luego recurrir a la compensación mediante explicación. Sin más preámbulo, véanse los siguientes ejemplos:

“红糖糍粑”: “ciba con azúcar morena” (ciba: pastelito frito de arroz glutinoso).

Obviamente, se vierte el “糍粑” como "ciba”, utilizando directamente su chino latinizado, y en el paréntesis se lleva a cabo la compensación explicando de qué se trata este alimento para que los lectores se formen una idea relativamente clara.

“木耳肉片”: “muer con cerdo” (muer: literalmente “oreja de árbol”: auricularia auricula-judae, hongo comestible en forma de oreja que crece en la corteza de los árboles) (Ramírez, 2004).

Con objeto de compensar la pérdida causada por el elemento muer, hay que optar por dejar claro explicando qué es y cómo es.

\section{Compensación mediante descripción}

Como se ha señalado, en la traducción de los nombres de los platillos, cuando los lectores de la lengua de destino no son capaces de comprender en general qué van a probar con la versión traducida de manera directa, utilizamos la compensación mediante explicación, recuperando sólo la parte difícil de en- 
tender, pero en el caso de que no tengan ni la menor idea de en qué consiste el platillo al leer la traducción directa, hace falta otra clase de técnica, que exploraremos en la presente sección.

Al traducir el platillo “霸王别姬” (literalmente: el mariscal despidiéndose de la concubina), conservar el valor estético traduciendo al pie de la letra o reflejar con especificación en qué consiste, depende en su mayoría de lo que postula la circunstancia. Si en cierta ocasión se requiere presentar el contenido de este platillo que se denomina de significado metafórico, recurriremos a la compensación mediante descripción. Cuando los lectores no comprendan las relaciones entre los platillos y su significado literal, será aconsejable utilizar esta técnica empleando el proceso llamado explicitación, y con él, como su denominación lo indica, "se expresa en la lengua terminal lo que está implícito en el contexto de la lengua de origen" (Vázquez, 1977). A continuación lo vamos a explicar a través de ejemplos:

“霸王 「姬”: “el mariscal despidiéndose de la concubina” (sopa de tortuga ${ }^{5}$ y pollo).

Para los comensales chinos parece lógico si no se pone de manifiesto en qué consiste este platillo. No obstante, de no aclarar la información implícita, el mensaje resultará oscuro o impreciso a primera vista para los hispanohablantes. A la luz de las opiniones de los traductólogos:

\section{[...] tales elementos pueden estar implícitos ya por razones de hábitos lingüísti- cos individuales, de características propias de una lengua, o ya por motivos de familiaridad del receptor del mensaje original con los aspectos de su cultura o con la experiencia que se transmite (Vázquez Ayora: 1977).}

Por ende, para dar mayor claridad hace falta dar complemento a través de la descripción del platillo después del significado literal, lo cual contribuye a que la versión surta el mismo efecto que el idioma original. A fin de familiarizarnos aún más con la "compensación mediante descripción", veamos más ejemplos donde se la utiliza:

5. Una especie de tortuga de caparazón blando que se utiliza en China para la cocina y la medicina. 
“蚂蚁上树”: “hormigas sobre el árbol” (fideos con picados de carne).

“红烧狮子头”: “cabezas de león cocidas con salsa de soya" (albóndigas cocidas con salsa de soya).

“龙虎斗”: “lucha de dragón y tigre” (sopa de serpiente y gato-leopardo).

“四大金刚”: “los cuatro reyes celestiales" (pan plano, churro chino, arroz glutinoso frito, leche de soya).

En resumen, en algunas ocasiones es preciso explicitar describiendo elementos que se encuentran implícitos en el contexto de la lengua original, a fin de realizar la compensación de las pérdidas y evitar un vacío en la comunicación del mensaje.

\section{Conclusión}

A pesar de que el tipo textual de la lengua de partida determina los estándares y las estrategias de traducción, la fidelidad, la comunicación y la elegancia siempre han sido lo que busca el traductor. En relación con el ámbito que tratamos en el presente estudio, deberíamos conceder primacía a la fidelidad, a fin de ofrecer a todo el mundo una visión verdadera de la gastronomía china. Y podríamos pretender dotar la traducción de la elegancia para presentar la sabiduría inherente a la denominación de los platillos chinos con base en la comunicación, un proceso que a veces nos cuesta llevar a cabo por el impedimento de las pérdidas surgidas. Por consiguiente, recurrimos a la compensación que, en fin, "trata de restablecer el equilibrio textual producido por las inevitables pérdidas y ganancias semánticas de la traducción” (Torre, 1994). Los principios de compensación concernientes a nuestro tema son los siguientes:

- Se utiliza la compensación cuando los contenidos perdidos desempeñan un papel clave en la comprensión del platillo.

- La información compensada tiene que ser concerniente a los contenidos correspondientes del nombre chino.

- Se concede prioridad a los contenidos perdidos más relevantes cuando surgen dos o más pérdidas.

- No importa qué medios se utilizan para recuperar las pérdidas, lo que realmente importa es conseguir una equivalencia dinámica, es decir, ponemos en un lugar primordial, en la presente tesis, el objetivo de hacer entender al lector en qué consiste el platillo. 
Al proceder a traducir los nombres de los platillos chinos, en cualquier circunstancia hay que seguir los principios precedentes cuidando la fluidez y la naturalidad en la reexpresión. Teniendo en cuenta todo lo arriba mencionado, procedemos a intentar ofrecer algunos consejos de utilidad en la traducción:

Antes de abordar la versión, el traductor debería tener una idea clara de los ingredientes, las formas de cocinar, así como del significado metafórico o tradición que contiene del platillo.

Ya que cualquier forma de traducir dará origen a algunas pérdidas, el traductor debería escoger la traducción más efectiva cuando el nombre original cuente con más de una versión posible. Por ejemplo: a causa de la flexibilidad de la traducción, además de "taco chino", preferiríamos indicar el “春卷” como “rollito de primavera” si ponemos énfasis en su relación con la Fiesta de la Primavera.

Conforme al principio de enfoque, el traductor debería recuperar la pérdida más relevante en cierta circunstancia, puesto que en algunas ocasiones al compensar una pérdida, se producirán otras. A manera de ilustración, tomemos de nuevo el ejemplo de “霸王别姬”: frente a más de una pérdida, optamos por compensar con "sopa de tortuga y pollo" por medio de la técnica de explicitación, en vez de relatar la historia de "el mariscal despidiéndose de la concubina”, una pérdida ocasionada por las disparidades históricas, porque para el lector el mensaje más importante es en qué consiste el platillo.

El traductor debería tener siempre en cuenta que la técnica de compensación más adecuada varía según el tipo de texto del que forman parte los nombres de los platillos. La carta del restaurante prefiere que los nombres traducidos reflejen con fidelidad en qué consiste el platillo con escasas palabras debido al espacio limitado, mientras que cuando se emplean los términos procedentes de una novela, se puede aclarar con todo detalle si la situación lo permite (utilizando la nota, por ejemplo) e incluso perseguir la elegancia.

En todo caso, al compensar las pérdidas en la traducción de los nombres de los platillos chinos, el traductor tiene que esforzarse dentro de lo posible por transmitir a los lectores la totalidad de los efectos de la lengua original. 


\section{Referencias bibliográficas}

García, V. (1989). En torno a la traducción: Teoría, crítica, historia. Madrid: Gredos ( $2^{a}$ edición corregida y aumentada).

Molina, L. (2006). El otoño del pingüino: Análisis descriptivo de la traducción de los culturemas. Castelló de la Plana: Universitat Jaume.

Nida, E. A. (2001). Language and culture: Contexts in translating. Shangai: Shanghai Foreign Language Education Press.

Ramírez, L. (2004). Manual de traducción chino / castellano. Barcelona: Gedisa.

Torre, E. (1994). Teoría de la traducción literaria. Madrid: Síntesis.

Vázquez, G. (1977). Introducción a la traductología. Estados Unidos: Georgetown University Press.

Xia, T. (2006). Estudio de la compensación en la traducción. Wuhan, Hubei: Education Press. 